\title{
Disostose Cleidocraniana: relato de caso em lactente
}

\author{
Cleidocranial Dysostosis: case report in infant
}

Paulo Víctor Innocencio Póvoa de Castro ${ }^{\dagger *}$, João Pedro Innocencio de Castro ${ }^{\dagger}$, Carlos Eduardo Rocha Pinto ${ }^{\dagger}$, Breno Souza Brito ${ }^{\dagger}$, Christianne Terra de Oliveira Azevedo

Como citar esse artigo. De Castro,

P.V.I.P; De Castro, J.P.I; Pinto,

C.E.R; Brito, B.S; Azevedo, C.T.O.

Disostose Cleidocraniana: relato de caso em lactente. Revista de Saúde. 2020 Jul./Dez.; $11 \quad$ (2): $29 \quad$ - 32.

\section{Resumo}

A Disostose Cleidocraniana é uma doença rara do tecido ósseo, a qual possui um padrão de herança genética autossômica dominante. Suas principais alterações são aplasia e hipoplasia clavicular, atraso no fechamento de suturas e fontanelas, presença de dentes supranumerários e retardo na erupção dos dentes permanentes. Essas alterações afetam negativamente a qualidade de vida de seus portadores por acarretarem danos à estética facial e corporal, à fonação e a função mastigatória. Este artigo tem o objetivo de apresentar o caso de uma lactente portadora de Disostose Cleidocraniana, analisando suas características clínicas e radiológicas de acordo com a literatura existente, apresentando ainda os possíveis tratamentos envolvidos no manejo da patologia. Lactente de 6 meses de idade, sexo feminino, cor branca, foi levada por sua mãe para consulta de puericultura. Apresenta histórico familiar para Disostose Cleidocraniana pelo lado materno, sendo que mãe, avó e tio são portadores da doença. Ao exame físico foram observados achados sugestivos de aplasia bilateral clavicular, hipertelorismo mamilar e microretrognatia. As fontanelas anterior e posterior encontravam-se abertas. Baseado no quadro clínico e histórico familiar, foi levantada a hipótese diagnóstica de Disostose Cleidocraniana. Ainda na consulta, a mãe apresentou uma radiografia de tórax da lactente, a qual confirmou a aplasia bilateral clavicular. Os dados clínicos e radiológicos atrelados ao histórico familiar positivo confirmaram a hipótese diagnóstica de Disostose Cleidocraniana. $\mathrm{O}$ diagnóstico e intervenção precoces possibilitam a promoção de um melhor desenvolvimento funcional, estético e uma melhor qualidade de vida para o paciente evitando possíveis sequelas funcionais, sociais e psicológicas.

Palavras-chave: Disostose Cleidocraniana, Lactente, Diagnóstico precoce.

\begin{abstract}
Cleidocranial Dysostosis is a rare autosomal dominant syndrome that affects the bone tissue. Its main alterations are clavicular aplasia and hypoplasia, closing delay of fontanelles and sutures, supernumerary teeth and an eruption delay of the permanent teeth. These changes negatively affect the quality of life of their patients as they cause damage to facial and body aesthetics, phonation and masticatory function. This article aims to present a case of an infant carrier of Cleidocranial Dysostosis, analyzing each clinical and radiologic features according to the existing literature, showing off possible treatments involved in the management of the patology. A 6 months old nursling, female, white, was taken by her mother to a childcare query. Presents a familiar history for Cleidocranial Dysostosis on the maternal side of the family, being possible to notice in view that her mother, grandmother and uncle are carriers of this pathology. On physical exam was possible to evidence suggestive findings as bilateral aplasia of clavicle, hypertelorism and micrognathism. Both anterior and posterior fontanelles were opened. Based on clinical and family history the hypothesis of Cleidocranial Dysostosis was made. The child's mom brought a radiography of her daughter's chest, confirming bilateral aplasia of the clavicles. All the findings in radiological and clinical exams added up to positive familiar history confirmed the diagnostic hypothesis of Cleidocranial Dysostosis. Early diagnosis and intervention enable the promotion of better functional, aesthetic development and a better quality of life for the patient, avoiding possible functional, social and psychological sequelae.
\end{abstract}

Keywords: Cleidocranial Dysostosis, Infant, Early diagnosis.

\section{Introdução}

A Disostose Cleidocraniana (DCC) é uma doença rara do tecido ósseo, a qual possui um padrão de herança genética autossômica dominante ${ }^{1,2}$. Apresenta uma prevalência estimada de 1:1.000.000 no mundo, não havendo predileção por sexo ou raça ${ }^{3}$.
Marie e Sainton, em 1898', criaram o nome "Disostose Cléidocrânienne Héréditaire" para essa condição. Atualmente, sabe-se que essa patologia afeta tanto os ossos de origem intramembranosa quanto os ossos de origem endocondral, sendo caracterizada como uma desordem esquelética generalizada ${ }^{2}$.

Pesquisas genéticas mapearam como causador

\footnotetext{
Afiliação dos autores:

${ }^{\dagger}$ Discente do Curso de Medicina da Universidade de Vassouras, Vassouras, RJ, Brasil.

${ }^{\ddagger}$ Docente do Curso de Medicina da Universidade de Vassouras, Vassouras, RJ, Brasil.

* Email de correspondência: pv.castro@hotmail.com
} 
desta enfermidade alterações no gene Cbfal (também nomeado de RUNX2), presente no braço curto do cromossomo 6p21, cuja função principal é a diferenciação osteoblástica, responsável por formar o tecido ósseo endocondral, intramembranoso e pela maturação dos condrócitos ${ }^{4}$.

A DCC é uma síndrome com características clínicas bem definidas, sendo seu diagnóstico feito usualmente através da avaliação clínica e radiográfica ${ }^{5}$. Suas principais alterações são aplasia ou hipoplasia clavicular, que são bilaterais em $80 \%$ dos casos, atraso no fechamento de suturas e fontanelas, presença de dentes supranumerários e retardo na erupção dos dentes permanentes ${ }^{6}$. Além das anormalidades relatadas acima, podem estar presentes ainda nessa síndrome: retenção de dentes decíduos, baixa estatura, tórax em sino, ossos wormianos, braquidactilia com falagens distais hipoplásicas, hipoplasia da pelve com fechamento tardio e sínfise púbica alargada, microretrognatia e hipertelorismo mamilar?

As alterações presentes nessa patologia a longo prazo afetam a qualidade de vida dos pacientes e acarretam danos funcionais, sociais e psicológicos por afetarem a estética facial, corporal, fonação e a função mastigatória ${ }^{2}$. $\mathrm{O}$ que ressalta a necessidade do diagnóstico precoce afim de evitar tais danos ${ }^{1}$.

Baseado nisto, este artigo tem o objetivo de apresentar o caso de uma lactente portadora de DCC, analisando suas características clínicas e radiológicas de acordo com a literatura existente, apresentando ainda os possíveis tratamentos envolvidos no manejo da patologia, buscando corroborar para um maior conhecimento científico sobre esta doença.

\section{Relato de caso}

Lactente de 6 meses de idade, sexo feminino, cor branca, procedente do município Três Rios-RJ, foi levada por sua mãe para consulta de puericultura. Os dados da anamnese foram colhidos com a mãe da lactente. Dados maternos: Realizado pré-natal completo (6 consultas) e sem intercorrências, negou a ingesta de bebidas alcoólicas e tabagismo durante o período de gestação, é portadora de Disostose Cleidocraniana.

Lactente nasceu à termo,com 40 semanas e 4 dias de idade gestacional, adequada para idade gestacional, parto vaginal induzido, banhada em mecônio. Apgar de 3 no $1^{\circ}$ minuto e de 7 no $5^{\circ}$ minuto. Ao nascimento, peso: 2.480 gramas, comprimento: 46 centímetros, perímetro cefálico: 32 centímetros. Apresenta histórico familiar positivo para Disostose Cleidocraniana pelo lado materno, sendo que mãe, avó e tio são portadores da doença. No momento da consulta, apresentava desenvolvimento neuropsicomotor e pôndero-estatural

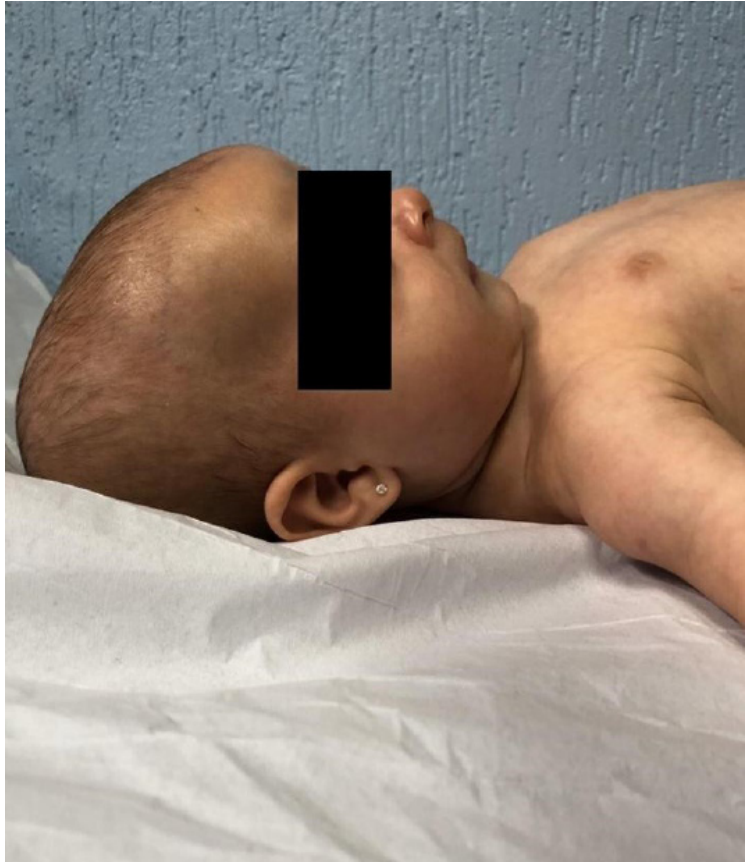

Figura 1. Face da lactente em perfil evidenciando microretrognatia.

normal para a idade, peso: 5210 gramas, comprimento: 61 centímetros, perímetro cefálico: 40 centímetros. Ao exame físico, foram observados achados sugestivos de aplasia bilateral clavicular, hipertelorismo mamilar e microretrognatia (figuras 1 e 2). As fontanelas anterior e posterior encontravam-se abertas. Baseado no quadro clínico e histórico familiar, foi levantado a hipótese diagnóstica de DCC. Ainda na consulta, a mãe apresentou uma radiografia de tórax da lactente, a qual confirmou a aplasia bilateral clavicular (figura 3 ).

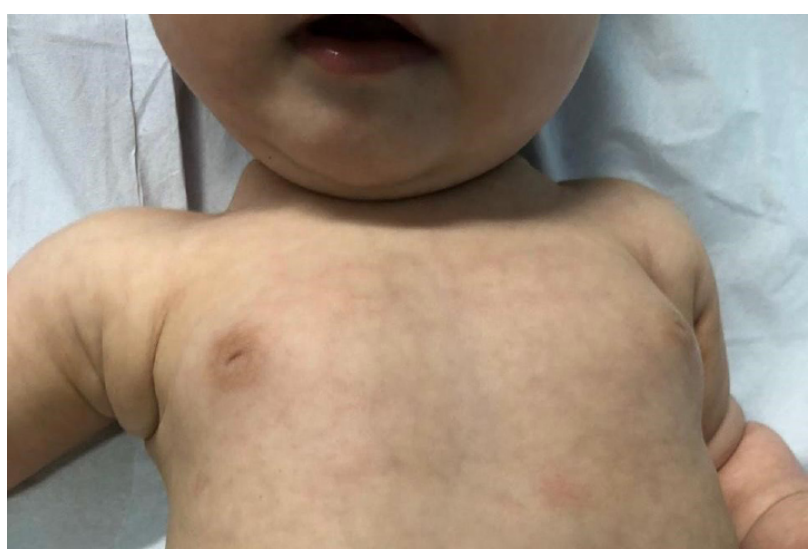

Figura 2. Exame físico do tórax com achados de hipertelorismo mamilar e aplasia clavicular.

Os dados clínicos e radiológicos atrelados ao histórico familiar positivo para DCC confirmaram a hipótese diagnóstica de Disostose Cleidocraniana. Foi orientado retorno mensal para avaliação da 
evolução neuropsicomotra e pôndero-estatural da lactente, e orientado o acompanhamento odontológico a partir da erupção dos dentes decíduos, bem como acompanhamento fonoaudiológico.

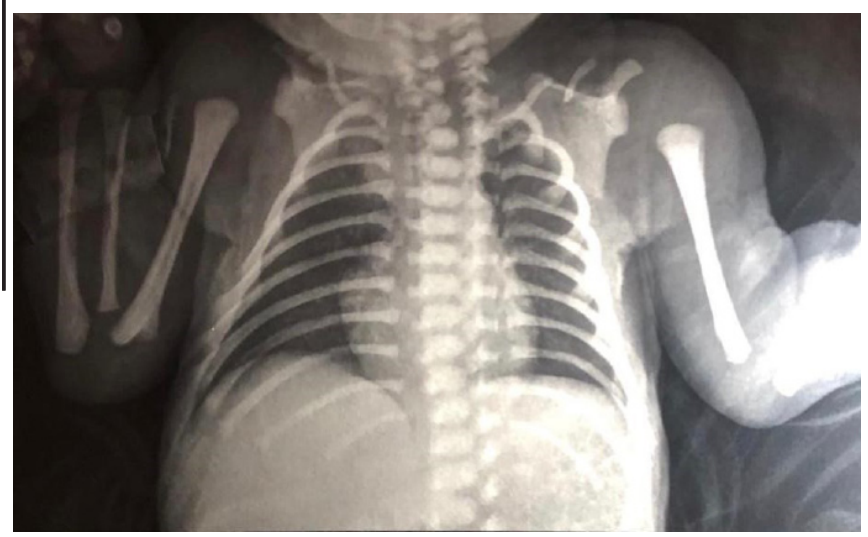

Figura 2. Exame físico do tórax com achados de hipertelorismo mamilar e aplasia clavicular.

\section{Discussão}

A DCC é uma desordem esquelética rara com uma prevalência notavelmente baixa, porém suspeitase que esse distúrbio seja subdiagnosticado ${ }^{8}$. Um fato que colabora diretamente com essa suspeita é devido a grande parte de pacientes com esta patologia não apresentar complicações físicas graves que contribuam para o aumento da mortalidade desse grupo ${ }^{1,8}$.

Sabe-se que essa desordem esquelética apresenta um padrão de herança genética autossômica dominante ${ }^{1}$. Esse fato pode ser sugerido no relato, uma vez que a lactente apresenta histórico familiar positivo pelo lado materno, apresentando um total de 3 casos na família, sendo que apresentam relação direta de parentesco, sem pular gerações (mãe, tio e avó).

Suas principais alterações clínicas são aplasia ou hipoplasia clavicular, atraso no fechamento de suturas e fontanelas, presença de dentes supranumerários e retardo na erupção dos dentes permanentes ${ }^{6}$. Os dados clínicos apresentados pela lactente vão de encontro com a literatura existente. Quanto as alterações dentárias, estas não podem ser avaliadas neste momento devido a idade da paciente, porém serão avaliadas em um segundo momento com o crescimento da mesma para corroborar com o diagnóstico.

Estudos relataram que a microretrognatia e o hipertelorismo mamilar são alterações clínicas presentes na $\mathrm{DCC}^{7}$. Durante o exame físico da lactente foram detectadas ambas as alterações, o que reforça a associação dessas características clínicas com a patologia.

Segundo a literatura o diagnóstico de DCC é eminentemente clínico e radiológico, sendo as radiografias de tórax e panorâmica da arcada dentária as imagens mais importantes ${ }^{1}$. No caso apresentado, observou-se as características clínicas típicas da doença, tais como hipoplasia clavicular bilateral e o atraso no fechamento da fontanela posterior. Somado a isso temos o histórico familiar positivo e a radiografia de tórax da lactente evidenciando a aplasia bilateral clavicular. Foi optado por não solicitar a radiografia panorâmica da arcada dentária levando em consideração o benefício mínimo deste exame em relação a idade da paciente e a dificuldade de execução do mesmo em um lactente, porém este exame será solicitado em um segundo momento durante futuras reavaliações da paciente.

Os pacientes com DCC evoluem na maior parte das vezes sem grandes complicações médicas, devido a isso a maioria dos diagnósticos são feitos tardiamente ${ }^{2}$. A consequência do diagnóstico tardio é a formação de um quadro grave de múltiplas alterações bucodentais, sendo elas: dentes supranumerários e impactados, erupção e/ou localização ectópica dos dentes ${ }^{9}$. No caso apresentado, foi possível a realização do diagnóstico precoce da patologia, o que contribui diretamente para um melhor desfecho da qualidade de vida da paciente, visto que é possível uma intervenção e acompanhamento precoces, evitando que se evolua para um quadro mais grave.

A abordagem multiprofissional é um pilar importante para o tratamento da doença, envolvendo profissionais da área médica, odontológica, fonoaudiológica e apoio psicológico ${ }^{1}$. Os principais objetivos do tratamento e acompanhamento são a promoção de uma melhora funcional da capacidade mastigatória, da fonação e estética facial e bucal ${ }^{2}$. Por essa desordem apresentar alterações na estética facial, bucal e corporal, é muitas vezes causa de problemas psicológicos e baixa autoestima nesses pacientes ${ }^{1}$. Devido a isso, tornam-se evidente os benefícios do diagnóstico precoce desta patologia, evitando possíveis consequências funcionais e psicossociais. A abordagem multiprofissional junto ao acompanhamento e intervenção precoces colaboram para uma melhor qualidade de vida desses pacientes, possibilitando uma inclusão adequada no meio social, evitando que estes pacientes sejam vítimas de bullying ou que apresentem baixa autoestima, e colaborando para o pleno desenvolvimento funcional da capacidade mastigatória, fonação e estética.

Foi desenvolvido o protocolo de tratamento Odontológico para Disostose Cleidocraniana, onde se avalia o tipo de caso, idade, grau de intensidade, e outras comorbidades apresentadas pelo paciente ${ }^{2}$. E definindo assim, as etapas de tratamento através da dentição atual do paciente ${ }^{2}$. Baseado no protocolo foi orientado para a lactente uma conduta expectante, visto que ainda não ocorreu a erupção dos dentes decíduos, sendo orientado um acompanhamento odontológico e fonoaudiológico 
a partir da erupção dos mesmos e reavaliações mensais com o médico pediatra.

\section{Conclusão}

É evidente que a DCC é um distúrbio raro e desconhecido por muitos na prática médica. ${ }^{9}$ Portanto é importante para o diagnóstico correto, o conhecimento de suas características clínicas e radiológicas. Tal processo possibilita a intervenção precoce dessa enfermidade, contribuindo para que as medidas terapêuticas possam ser instituídas no momento adequado, visando justamente promover um melhor desenvolvimento funcional e estético e uma maior qualidade de vida para o paciente evitando possíveis sequelas funcionais, sociais e psicológicas. Reforça-se ainda a necessidade da abordagem multidisciplinar no manejo desta patologia.

\section{Referências}

1. Mundlos S. Cleidocranial Dysplasia: Clinical and Molecular Genetics. J Med Genet 1999; 36(3):177-82.

2. Dantas AG, Lima RAS, Ferreira MM, Leles GV, Vianna ACF. Displasia Cleidocraniana: diagnóstico através de exames de imagem e clínico. Rev Odont Contemp - ROC 2017; 1(2):82-91.

3. Tanaka JLO, Ono E, Filho EM, Castilho JCM, Moraes LC, Moraes MEL. Cleidocranial Dysplasia: Importance of Radiographic Images in Diagnosis of the Condition. J Oral Sci 2006; 48(3):161-6.

4. Wang XP, Fan J. Molecular genetics of supernumerary tooth formation. Gênese. 2011; 49(4):261-77.

5. El-Gharbawy AH, Peeden JN, Lachman RS, Graham JM, Moore SR, Rimoin DL. Severe Cleidocranial Dysplasia and Hypophosphatasia in a Child With Microdeletion of the C-terminal Region of RUNX2. Am J Med Genet A. 2010; 152a(1):169-74.

6. Toguel AV, Piepers I, de Wilde L. The significance of the clavicle on shoulder girdle function. J Shoulder Elbow Surg. 2015; 24(9):e255-9.

7. Golan I, Baumert U, Hrala BP, Müssig D. Dentomaxillofacial Variability of Cleidocranial Dysplasia: Clinicoradiological Presentation and Systematic Review. Dentomaxillofac Radiol. 2003; 32(6):347-54.

8. Cooper SC, Flaitz CM, Johnston DA, Lee B, Hecht JT. A Natural History of Cleidocranial Dysplasia. Am J Med Genet 2001; 104(1):1-6.

9. Bufalino A, Paranaíba LMR, Gouvêa AF, Gueiros LA, Martelli-Júnior H, Júnior JJ, et al. Cleidocranial Dysplasia: Oral Features and Genetic Analysis of 11 Patients. Oral Dis 2012; 18(2):184-90. 